\title{
Les milliaires tardifs, une réception particulière de l'autorité impériale. Un paysage particulier le long des voies de Lusitanie
}

\author{
Sabine Lefebvre ${ }^{1}$
}

Dans tout l'empire, alors que les princes se succèdent parfois très rapidement au cours du $\mathrm{III}^{\mathrm{e}}$ siècle mais aussi au IV ${ }^{\mathrm{e}}$ siècle, leurs noms et leurs titulatures sont particulièrement présents dans les espaces ruraux des cités, en figurant sur les milliaires. Alors que les voies ne sont pas forcément refaites sous chaque règne, on constate cependant qu'à certains emplacements - carrefours, ponts ...--, un grand nombre de milliaires, souvent proches dans le temps, sont placés et proposés au regard des voyageurs, constituant un paysage particulier. Quel intérêt $\mathrm{y}$-a-t-il à une telle concentration constituant parfois une forêt de pierres ? Et comment expliquer que sur certains de ces milliaires, la titulature du prince ne soit pas au nominatif mais au datif, prenant alors la forme d'un hommage rendu au détenteur du pouvoir ? Cette pratique, connue en Orient ${ }^{2}$, l'est aussi dans l'extrême Occident, dans la province de Lusitanie. Reflétant l'impact des changements au sommet de l'Etat, elle est la manifestation d'un déplacement du centre des villes aux zones rurales où les grandes familles possédaient uillae et grands domaines.

\section{Présentation de la problématique}

Si l'impact de Rome dans les centres urbains des provinces est aujourd'hui bien connu d'une part par la parure monumentale des villes qui comportent, autour des fora, basiliques, temples et curies ... et d'autre part par les inscriptions,

1 S. Lefebvre, Université de Bourgogne-Franche-Comté / UMR 6298 ARTEHIS: Je remercie Anne Gangloff pour sa relecture attentive. Pour alléger les notes, le lemme des inscriptions citées sera réduit à la référence la plus récente ou la plus utilisée, sauf en cas de discussion sur un point précis. Les références complètes peuvent être retrouvées soit sur le site de ClaussSlaby, soit sur le site d'Hispania epigraphica. Les abréviations mentionnées sont celles utilisées sur le site de Clauss-Slaby (http://db.edcs.eu/epigr/hinweise/abkuerz.html). Ces deux sites proposent, pour une grande partie des documents cités, des photographies.

2 Cf. T. Bauzou, <Epigraphie et toponymie: le cas de la Palmyrène du sud-ouest >, Syria 70 (1993), 27-50.

(C) SABINE LEFEBVRE, 2022 | DOI:10.1163/9789004411449_008

This is an open access chapter distributed under the terms of the CC BY-NC 4.o liçense. 
dont les hommages rendus aux princes de leur vivant ou après leur divinisation $^{3}$ sont un exemple - c'est alors un paysage urbain très particulier qui se constitue progressivement -, cet impact peut sembler moindre dans les espaces ruraux. Or, la présence des uillae, résidences des notables vivant entre le centre politique de leur ciuitas et leur domaine agricole, témoigne que cet impact était important: par les techniques utilisées et par leur décor, on perçoit bien que le modèle romain s'est largement diffusé. Ces uillae sont ainsi un centre de diffusion de la culture romaine, mais aussi des pratiques sociales et politiques ${ }^{4}$.

Mais cet impact est aussi bien visible par le soin apporté au réseau routier par les empereurs, réseau qui fut régulièrement restauré, aménagé, y compris dans les confins de l'empire comme en Lusitanie ${ }^{5}$. Pouvant être datés grâce aux milliaires découverts le long des voies et où figure le nom de l'empereur, au nominatif en général, ces travaux et leur trace écrite permettaient aux ruraux d'être en contact avec le pouvoir impérial, contact modeste certes, mais réel. De fait, le paysage visible depuis la route par l'usager reflète la politique impériale $^{6}$ - c'est un paysage de réception d'un message politique -, sans qu'une dimension esthétique soit donnée à cette concentration de pierres bordant la voie : il s'agit par ces implantations, de tenir les provinciaux au courant des modifications au centre du pouvoir et des actions décidées, éventuellement par les princes. Ce paysage, fort différent de celui proposé dans les centres urbains où la monumentalisation de l'image impériale est de mise, est néanmoins fort intéressant à analyser.

On constate en effet qu'à partir du milieu du III ${ }^{\mathrm{e}}$ siècle et au $\mathrm{IV}^{\mathrm{e}}$ siècle, ces milliaires restent très fréquents, même en période de crise politique, et, parfois,

3 S. Lefebvre, < Hommages rendus au Divus Augustus en Occident, témoignages d'une relation particulière? ?, dans S. Lefebvre et A. Daguet-Gagey (éds.), L'empereur Auguste et la mémoire des siècles (Arras 2018), 123-168; S. Lefebvre, < Rendre hommage aux princes morts Nerva et Trajan. Les Divi comme facteur d'enracinement de la nouvelle dynastie >, dans A. Caballos Rufino (éd.), De Trajano à Adriano. Roma matura, Roma mutans (Séville 2019), 143-167.

4 On peut voir J.-G. Gorges, $<$ Villes et villas de Lusitanie (Interactions, échanges, autonomies) >, dans J. de Alarcão et al. (éds.) Les Villes de Lusitanie romaine. Hiérarchies et Territoires. Table ronde du CNRS (Talence, le 8-9 novembre 1988) (Paris 1990), 91-113, en part. 107 : « De fait, villes et routes de Lusitanie rassemblent dans leur sphère d'influence directe la majeure partie des établissements ruraux répertoriés. Près de $80 \%$ des villas recensées sont situées dans un rayon allant de 5 à $30 \mathrm{~km}$ autour des villes ou s'échelonnent le long des axes routiers, à une distance moyenne variant entre 1 et 10 kilomètres ».

5 S. Marcos, <Espace géographique, espace politique. La frontière provinciale lusitanienne, une limite déterminante? >, dans A. Caballos Rufino et S. Lefebvre (éds), Roma generadora de identidades. La experiencia hispana (Madrid 2011), 171-184.

6 On pourrait évoquer ici un < paysage politique >, dans un contexte particulier, celui des crises au sommet de l'État, qui conduit à son évolution constante au fur et à mesure de la succession des empereurs. 
leur formulation est modifiée : la titulature du prince n'est plus au nominatif mais au datif, comme dans les hommages rendus dans les espaces publics de la cité7.

Il s'agit de montrer comment les voies deviennent un nouvel espace de propagande impériale, comment les hommages publics sis jusque-là dans les centres urbains, gagnent les espaces ruraux, changeant subtilement le paysage visible depuis les voies : les bornes milliaires ne sont plus uniquement des marqueurs de distances, elles deviennent des supports de la communication des princes, des milliaires-hommages. Je me propose d'analyser quelques dossiers provenant de Lusitanie, illustrant cette pratique.

\section{Le réseau routier et les milliaires de Lusitanie}

Les cartes dressées par nos collègues 8 témoignent de la forte présence de ces milliaires, entre autres en Lusitanie, mis en place durant plus de quatre siècles : ils constituent, le long des voies, des repères, des marqueurs qui participent à l'élaboration d'un paysage particulier, vécu ${ }^{9}$, celui qui est visible depuis la route mais aussi depuis les uillae, ateliers, nécropoles qui la bordent. Constitutifs de la uia, ils rythment son parcours, proposant une vision qui se répète de mille en mille. Ainsi que le rappelle J.M. Roldán Hervás ${ }^{10}$, « el proceso de colocación de miliarios fue acumulativo, es decir, se fueron añadiendo de forma sucesiva, incluso en un mismo punto, sin retirar los anteriores». Beaucoup ont subi les outrages du temps et nombre ont été déplacés, de quelques centaines de mètres à l'époque médiévale ou de plusieurs kilomètres, transformés en colonnes pour des maisons ou des églises. Il est donc souvent difficile de

7 S. Lefebvre, < Les critères de définition des hommages publics en Occident 〉, BAntFr 1998 (2002), 102-113.

8 J.-Ma. Solana Sainz et L. Sagredo San Eustaquio, La política viaria en Hispania. Siglo IV D.C. (Valladolid 1997); J.-Ma. Solana Sainz et L. Hernández Guerra, La política viaria en Hispania. Siglo III d.C. (Valladolid 2002); J.-Mª. Solana Sainz et L. Sagredo San Eustaquio, La red viaria romana en Hispania siglos I-IV d.C. (Valladolid 2006); J.-Ma. Solana Sainz et L. Sagredo San Eustaquio, La política viaria en Hispania siglos I-II d. C. (Valladolid 2008). On peut également mentionner le projet du CIL XVII, mais les volumes portant sur la Lusitanie ne sont pas encore parus.

$9 \quad$ P. George (éd.), Dictionnaire de la géographie ${ }^{2}$ (Paris 1974), 337-338: « [...] il a pris récemment une signification synthétique rassemblant l'ensemble des traits issus de la géographie naturelle et des apports accumulés des civilisations qui ont façonné successivement le cadre initial et sont entrés dans la conscience de groupe des occupants. Il devient synonyme d'environnement dans les processus de perception de l'espace - se confond alors avec l'espace vécu».

10 J.M. Roldán Hervás, < Los miliarios de la vía de la Plata >, dans Sociedad Estatal de Commemoraciones Culturales (éd.), La vía de la plata. Una calzada y mil caminos. Exposition au Museo Nacional de arte romano (Mérida, 21 février au 13 avril 2008) (Madrid 2008), 70-79, en part. 71. 


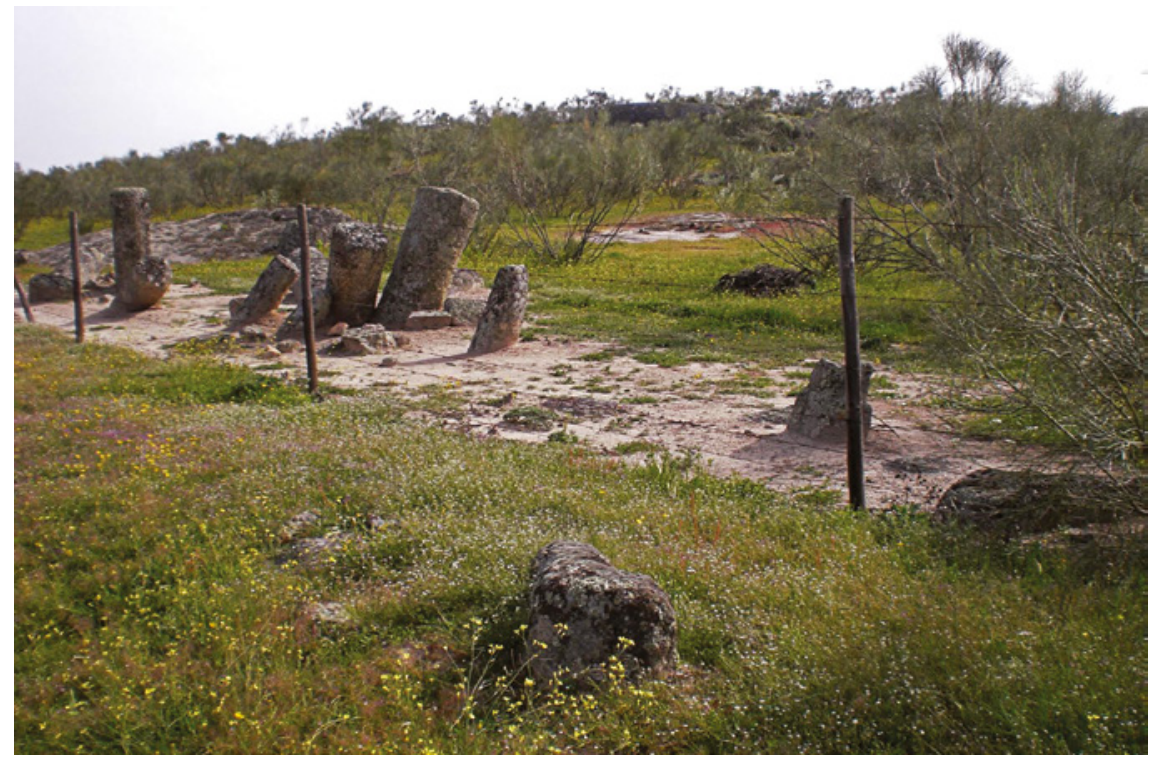

FIGURE 7.1 Milliaires de la Via de la Plata

PHOTOGRAPHIE ISSUE DE https://www.tripadvisor.com/LocationPhotoDirect Link-g2449867-d6403504-i238026701-Actividades_Barbancho-Casar_de_ Caceres_Province_of_Caceres_Extremadura.html

préciser leur emplacement initial, de restituer le paysage auquel ils participaient, et de localiser mansiones ou autres stationes figurant dans les Itinéraires à des milles précis, mais aussi de mettre en rapport leur emplacement initial avec leur contexte : sont-ils proches d'un petit noyau d'habitation, d'une uilla, d'une nécropole ... Ces données importantes, il est parfois difficile d'en disposer. Rares sont les lieux où sont encore en place les milliaires, proposant un paysage tout à fait particulier (fig. 7.1).

Nous ne disposons que d'une infime partie des milliaires mis en place, nombreuses devaient être les bornes de bois qui ont bien entendu disparu. Tous ces milliaires n'étaient pas gravés - certains étaient sans doute peints -, et la présence de l'écrit, gravé dans la pierre avait sans nul doute une signification, faisant d'une partie de ces paysages ruraux, une page d'écriture.

Rythmant le territoire rural des ciuitates, devenant des éléments organiques du paysage rural ${ }^{11}$, les milliaires sont, pour les ruraux, l'un des premiers

11 Mais d'un paysage de proximité, car les milliaires placés le long des voies devaient être lisibles. Ils constituent ainsi le premier plan des paysages que l'usager découvre au fur et à mesure de son avancée. Bien que très visibles, ils ne devaient cependant pas être systématiquement tous lus par chaque passant. On peut donc s'interroger sur leur rôle et leur impact réel. 
contacts $^{12}$ avec le prince au pouvoir, bien que tous les princes n'aient pas contribués à la réfection de chacune des voies de l'empire. Les cartes proposées par J.-Ma. Solana Sainz et L. Sagredo San Eustaquio ${ }^{13}$ mettent en évidence les routes ou tronçons sur lequel tel ou tel prince a mis l'accent. Ce contact avec l'empereur va prendre une importance cruciale quand les crises politiques se succèdent et que la nécessité de mener une propagande active se fait de plus en plus sentir. Les répercussions de l'action impériale vont alors être ressenties non seulement dans les centres urbains, qui accueillaient déjà, au sein des forums, les messages émanant de Rome, mais aussi dans l'espace rural, afin de toucher le maximum de sujets. Le support choisi, qui va alors être privilégié, est le milliaire; familier dans le paysage, il permet d'allier utilité et propagande. Utilité certes, car il rythme la voie, mais une utilité relative, d'une part car il semble, vu leur proximité dans le temps, que peu soient liés à des travaux de réfection, et d'autre part, car positionnés en des lieux où se trouvaient déjà d'autres milliaires, leur utilité pour indiquer les distances était redondante. C'est donc bien la propagande impériale qui semble ici avoir conditionnée leur mise en place, avec une forme inhabituelle de la titulature impériale, au datif.

\section{3}

\section{La uia XXIV}

Le dossier que j'ai retenu pour illustrer cette pratique est celui de la uia XXIV, la «Via de la Plata ${ }^{14} »$, née à Augusta Emerita et qui relie Asturica Augusta ${ }^{15}$ avec, entre ces deux villes, 313 milles. Elle traverse le territoire de plusieurs cités $^{16}$, entre autres Augusta Emerita, Norba, Caurium ${ }^{17}$, Capera, Salmantica et se poursuit en Citérieure. Il s'agit d'un des axes principaux de la péninsule Ibérique, tant sur le plan économique que stratégique (fig. 7.2): en témoignent

12 L'autre contact se fait via le monnayage.

13 Solana Sainz et Sagredo San Eustaquio 2006, op. cit. (n. 8).

14 J.M. Roldán Hervás, Iter ab Emerita Asturicam. El camino de la plata (Salamanque 1971); Sociedad Estatal de Commemoraciones Culturales 2008, op. cit. (n. 10).

15 Elle se prolonge jusqu'à Caesaraugusta; cf. Itin. Anton. 433, 1 (ed. de O. Cuntz, Itineraria Romana, I [Stuttgart 1990], 67).

16 Il est très difficile en péninsule Ibérique de déterminer les limites précises des cités; la plupart des tracés s'appuient sur les limites naturelles, rivières ou hauteurs. Cf. E. Cerrillo Martín de Cáceres, J. M. Fernández Corrales et G. Herrera García de la Santa, < Ciudades, territorios y vías de comunicación en la Lusitania meridional española >, dans J. de Alarcão et al. 199o, op. cit. (n. 4), 51-72, en part. 57, fig. 3.

17 La limite orientale de Caurium, frontière occidentale de Capera est formée par « una línea oblicua equidistante entre ambas ciudades que iría desde la Sierra de Gata hasta la de Cañaveral (que) sirve como separacíon artificial por oriente » selon J.E. Ortega, Corpus de inscripciones latinas de Cáceres IV. Caurium (Cáceres 2016), 9. 


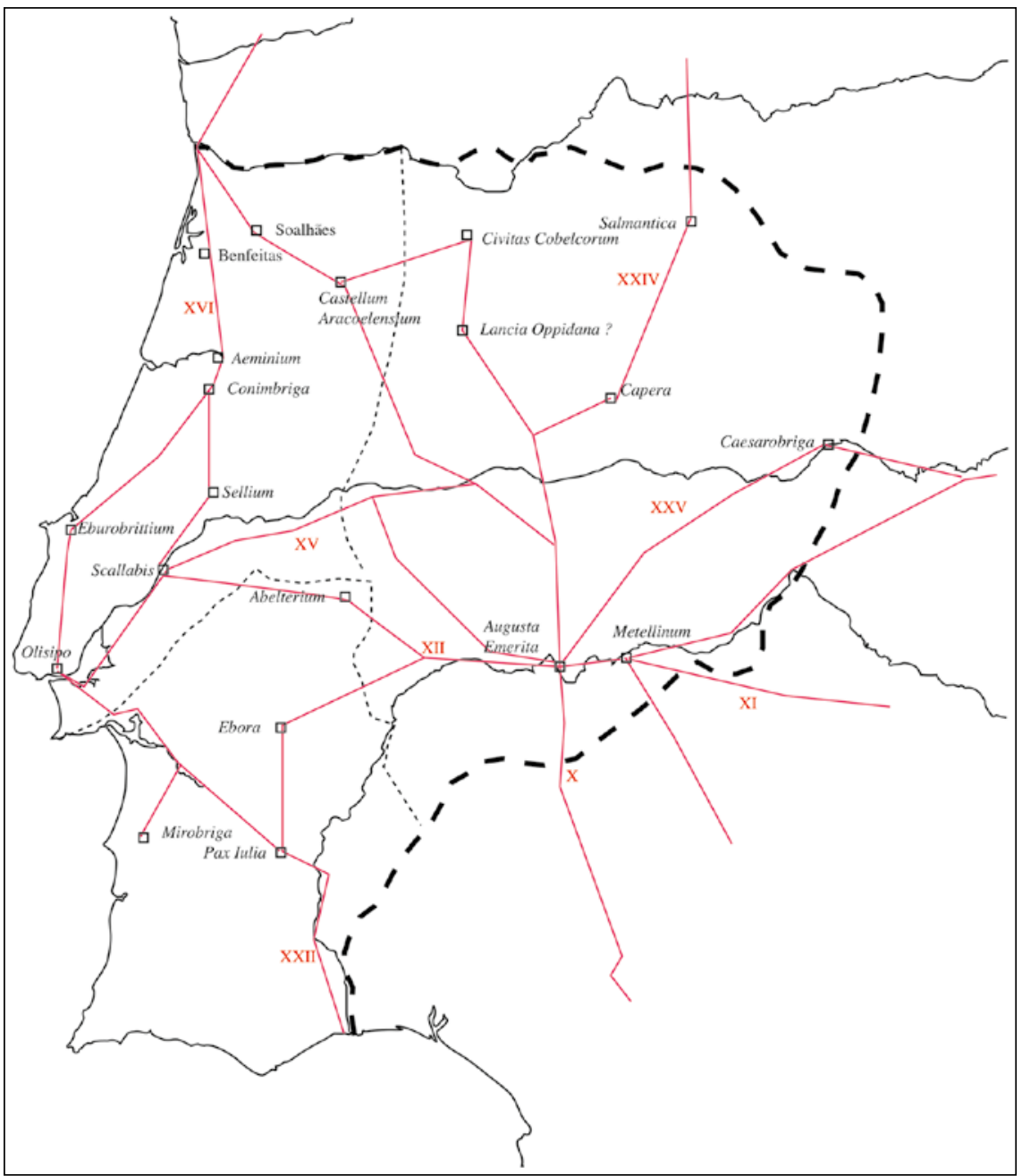

FIGURE 7.2 Les principales voies de Lusitanie RÉALISATION S. LEFEBVRE D'APRÈS LEFEBVRE 2016, OP. CIT. (N. 23), 270

les ouvrages d'art comme le pont d'Alcantara ou celui de Salamanque. Les milliaires qui la bordent, datés du I ${ }^{\mathrm{er}}$ au $\mathrm{IV}^{\mathrm{e}}$ siècles $^{18}$, d'Auguste à Valentinien $\mathrm{I}^{\mathrm{er}}$, témoignent de l'intérêt permanent des princes pour cet axe fondamental de circulation. La majorité des milliaires découverts - 198 décomptés en $1995^{19}$

18 Roldán Hervás 1971, op. cit. (n. 14), 47-71; depuis la publication de cet ouvrage, des découvertes ont été faites, ainsi que des relectures.

19 Cf. C. Puerta Torres, Los miliarios de la vía de la Plata, (thèse de doctorat, Madrid 2002, voir https://eprints.ucm.es/2439/, consulté le 26/3/2021) ce qui est assez conséquent ; voir aussi Roldán Hervás 2008, op. cit. (n. 10), 70-79, en part. 71. 
- se concentre sur un tiers environ du trajet, en territoire lusitanien ${ }^{20}$; la plupart ont été retrouvés in situ ou à proximité de la chaussée antique, permettant d'avoir une idée du paysage antique, ce qui est évidemment une chance, même s'il reste aujourd'hui difficile de les localiser précisément pour d'autres. Tous n'indiquent pas de distances, j'y reviendrai.

J'ai retenu un tronçon assez long de cette uia XXIV ${ }^{21}$, au-delà du territoire d'Augusta Emerita. Ce très gros centre urbain et capitale provinciale, puis capitale du diocèse des Espagnes, exerce une forte influence sur un espace plus vaste que son territoire civique et est très attractif ${ }^{22}$. Les pratiques, et en particulier la réception et l'impact des politiques impériales y étaient forcément différentes ${ }^{23}$. Je vais donc m'intéresser au tracé de la voie depuis le territoire de Norba (Cáceres) jusqu'à la frontière de la Lusitanie, au nord du territoire de Salmantica (Salamanque). J'envisagerai un focus plus particulier sur le territoire de Capera, en tenant compte des lieux où se trouvent des milliaires au datif ${ }^{24}$.

La mansio Ad Sorores ${ }^{25}$ est au sud de Norba, à 26 milles de Mérida. À proximité du site ${ }^{26}$, on a retrouvé des milliaires, et au $31^{\mathrm{e}}$ mille, à Aldea del Cano, un milliaire restitué au datif pour un empereur anonyme ${ }^{27}$. À $29 \mathrm{~km}$ au nord de Ad Sorores, on trouve la mansio Castris Caecilia ${ }^{28}$ sur le territoire de la cité de Norba $^{29}$, au nord de la ville de Cáceres, au mille 46 sans doute ${ }^{30}$. À proximité,

20 Roldán Hervás 1971, op. cit. (n. 14), 47 : il propose que la gestion de la uia devait être en lien avec le découpage provincial.

21 Cf. Annexe 1. La section lusitanienne de la Via de la Plata.

22 Gorges 1990, op. cit. (n. 4), 97-100.

23 S. Lefebvre, « Réception du pouvoir impérial en Lusitanie de Dioclétien à la fin de la dynastie constantinienne >, dans J. d'Encarnação, M. Conceição Lopes et Pedro C. Carvalho (éds.), A Lusitânia Romana - Entre Romanos e Bárbaros, VIII Mesa-redonda Internacional à Mangalde (Coimbra et Mangualde 2016), 223-279.

24 Ils figurent souvent dans des groupes de plusieurs milliaires antérieurs, constituant ainsi un paysage particulier (fig. 1).

25 Itin. Anton. 433, 3: m. p. XXVI (ed. Cuntz 199o, op. cit. [n. 15], 67); Ravenn. IV 45, 15: Sorores, quae confinatur cum (ed. J. Schnetz, Itineraria Romana, 2, Ravennatis anonymi cosmographia et Guidonis geographica [Stuttgart 199o], 82); Plin. Nat. 4.117: [...] coloniae Augusta Emerita, Anae fluuio adposita, Metellinensis, Pacensis, Norbensis Caesarina cognomine; contributa sunt in eam Castra Seruilia, Castra Caecilia. Cf. Roldán Hervás 1971, op. cit. (n. 14), 79-8o, dans le village de Casas de Don Antonio, plus précisément dans la dehesa de Santiago Bencáliz, domaine foncier municipal.

26 Sur le site, on a retrouvé des restes de bâtiments.

27 MiliariosPlata 39 Aldea del Cano: [---] / [--- $\max ($ imo $)$ [---], en remploi.

28 Itin. Anton. 433, 4: m. p. XX (ed. Cuntz 199o, op. cit. [n. 15], 67); Ravenn. IV 45, 14: Castris (ed. Schnetz 199o, op. cit. [n. 25], 82). Cf. Roldán Hervás 1971, op. cit. (n. 14), 8o-83.

29 Il est inutile ici de revenir sur les liens entre Castra Caecilia et Norba, en lien avec le texte de Pline l'Ancien (cf. n. 25).

Au niveau de Cáceres el Viejo. 
au nord, se trouve le village de Casar de Cáceres ${ }^{31}$ qui correspond au mille LII. Outre des milliaires du $\mathrm{II}^{\mathrm{e}}$ et du début du $\mathrm{III}^{\mathrm{e}}$ siècle, on y a découvert un milliaire de Constance Chlore au datif ${ }^{32}$ daté de 293-305, retravaillé après 305 .

Juste après le passage du Tage en venant du sud ${ }^{33}$, se trouve le site d'Alconetar qui comprend un fortin antique, une villa rurale complétée à l'époque tardo-antique par une basilique du $\mathrm{v}^{\mathrm{e}}$ siècle et un édifice funéraire ${ }^{34}$. Le lieu est éminemment stratégique ${ }^{35}$ et pourrait être assimilé à la mansio Turmulus ${ }^{36}$, même si un autre site, un peu plus au nord correspond mieux aux distances indiquées par l'Itinéraire d'Antonin ${ }^{37}$. Plusieurs inscriptions y ont été découvertes $^{38}$ - beaucoup en remploi dans le village de Garrovillas -, des funéraires ${ }^{39}$ mais surtout des milliaires de Tibère, Néron, Septime Sévère, Hadrien, Marc Aurèle ou Caracalla au nominatif, plusieurs milliaires anépigraphes, et au datif, un milliaire attribué à Caracalla ou à Probus, mais qui reste très douteux ${ }^{40}$ - il a disparu! -, un de Probus ${ }^{41}$ assuré, tous deux très proches l'un de l'autre près

$31 \quad$ Il se trouve à $9 \mathrm{~km}$ de Castra Caecilia.

32 MiliariosPlata 66 Casar de Cáceres:D(omino) $n$ (ostro) $F[l($ auio) $] /$ Vale [rio]/[Con[stantio], $/[n]$ ob(ilissimo) Ca[es(ari)]. Un second texte se superpose : $D$ (omino) $n($ ostro $)[\operatorname{Im}] p(e$ ratori) / Caes[ari] [---]V[---]R[---] / [[---]], / [n]ob(ilissimo) Ca[es(ari)]. La surcharge de Imperator sur Dominus noster est attestée en Bétique; cf. par exemple P. Sillières, Les voies de communication de l'Hispanie méridionale (Bordeaux 199o), 70-71, n. 4.

33 Territoire de la cité de Norba (Cacéres), limité au nord par le Tage. On peut s'étonner que Garrovillas soit localisé sur le territoire de Norba.

34 L. Caballero Zoreda, Alconetar en la vía romana de la Plata, Garrovillas (Cáceres) (Madrid 1970) ; E. Cerrillo Martín de Cácerez et A. Montalvo Frías, «La vía de la Plata en Extremadura de Augusta Emerita a Caecilius Vicus», dans Sociedad Estatal de Commemoraciones Culturales 2008, op. cit. (n. 10), 49-57, en part. 53 .

35 Caballero Zoreda 1970, op. cit. (n. 34), 3.

36 Itin. Anton. 433, 5 :m. p. XX (ed. Cuntz 199o, op. cit. [n. 15], 67); Ravenn. IV 45, 13: Turmulum (ed. Schnetz 199o, op. cit. [n. 25], 82). Cf. Caballero Zoreda 197o, op. cit. (n. 34), 8.

37 Roldán Hervás 1971, op. cit. (n. 14), 83-86.

38 Caballero Zoreda 1970, op. cit. (n. 34), Appendice II, 128-135. Sur le site Clauss-Slaby, on n'en décompte à ce jour que 26 , et 17 sur le site d'Hispania epigraphica qui n'a pas retenu les milliaires anépigraphes.

39 CILCaceres I, Garrovillas de Alconetar 200 ; 201; 202 ; 203 ?; 204; 205 ?; 206 ; 207 ?.

$40 \quad$ CIL II $6204($ HAEp 1807) = CPILCaceres $683=$ MiliariosPlata $91=$ CILCaceres I $210($ HEp 16, 96) Garrovillas de Alconetar: Imp(eratori) Caes(ari) / Marco [Aur(elio) [---] / P(io) [F(elici)] Inuic/to Augu[sto], / [---] près du pont d'Alconetar. Dans la lacune pourrait se trouver la mention du grand pontificat. Une autre lecture a été envisagée dans le $C I L$ pour ce milliaire aujourd'hui disparu: $\operatorname{Imp}($ eratori) Caes(aris) / M(arco) / p (io) Inuic/to duci / $\operatorname{maxi}(\mathrm{mo})$. Cette lecture est plus qu'improbable, en particulier pour les dernières lignes conservées.

41 MiliariosPlata 92 Garrovillas de Alconetar: $\operatorname{Imp}($ eratori $) C($ a)esar $[i] / M(\operatorname{arco})$ Aurel(io) Pro[bo], / P(io) Felici Inuicto, / [Augusto ---], au mille LXv. 


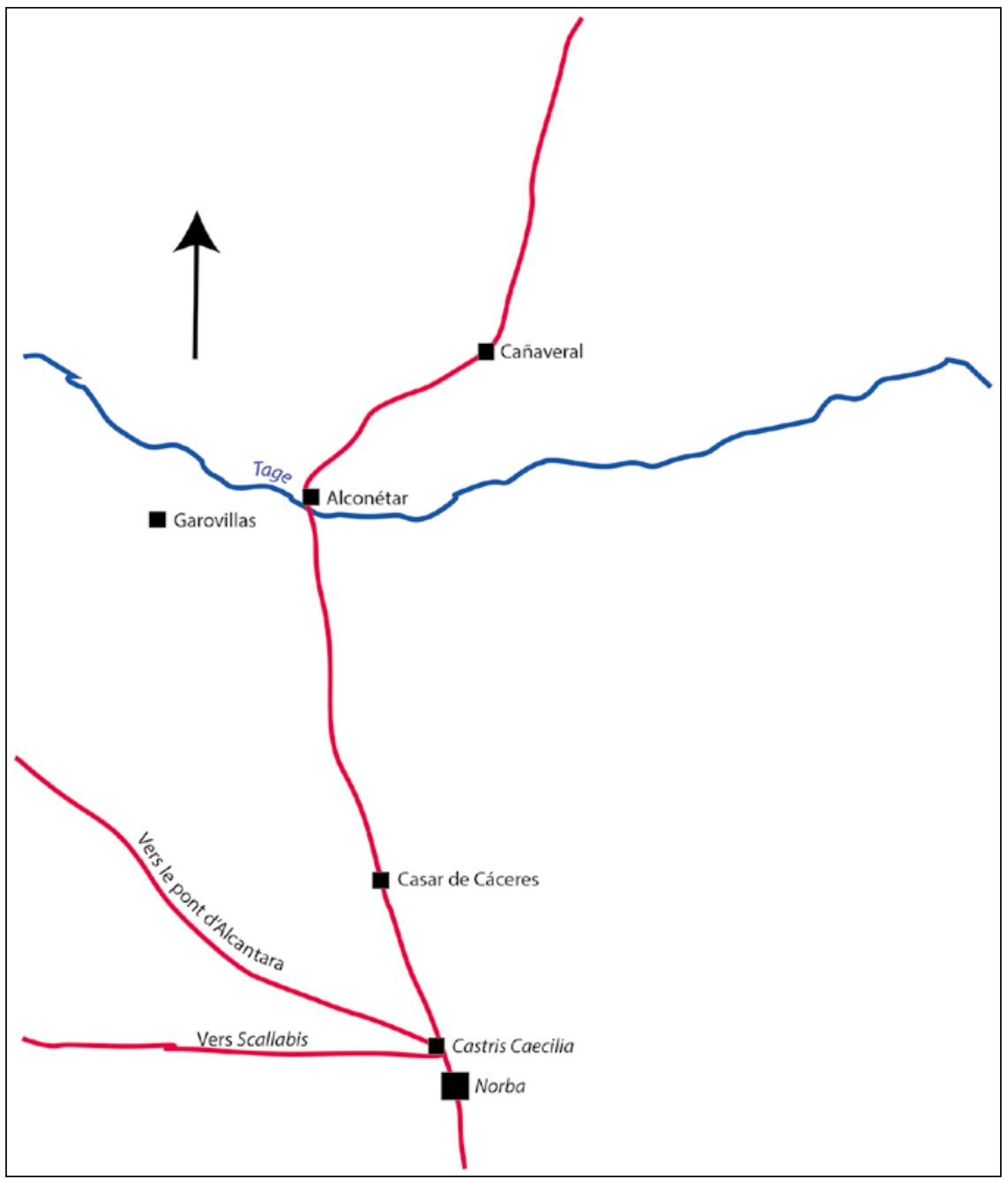

FIGURE 7.3 Localisation des mansiones Castris Caecilia et Turmulus

du pont d'Alconetar, et à Cañaveral, un milliaire de Constance II au datif ${ }^{42}$ daté entre 337 et 361 . Ce sont donc deux milliaires assurés au datif que l'on trouve à proximité de la mansio.

42 J. Río-Miranda et $\mathrm{M}^{\mathrm{a}} \mathrm{G}^{\mathrm{a}}$ Iglesias Domínguez, < Nuevas aportaciones a la epigrafía y arqueología de Cáceres >, Ahigal 24 (2005), 13, n. 3 (HEp 14, 86 = FE 6o2) = CILCáceres IV $1159=$ AE 2017, 654 Cañaveral : [Caesa]r (sic!) Iul(io) (sic!) / Co(n)stan/tio (sic!), trium/p(h)ator (sic!) $m[$ ax (imo)] (sic!), / [s]enper (sic!) A(u)gus(to) (sic!), /b(ono) r(ei) p(ublica) $n($ ato). 
En se dirigeant vers le nord, en direction de la ville de Capera, on trouve à Carcaboso, plusieurs milliaires en remploi dans l'église de la ville avec un «Parque de los miliarios ». Le remploi post-antiquité dans les édifices du village de Carcaboso génère une concentration sur un point qui n'existait pas dans l'antiquité. C'est une des difficultés que l'on rencontre lorsque l'on étudie les milliaires. C'est donc au sud du village actuel que le milliaire de Flavius Sévère au datif a dû être placé. Je n'ai pu retrouver d'informations sur les éventuels restes d'une uilla ou d'un établissement rural. Ce site est considéré comme appartenant à la cité de Capera, juste au sud de la ville romaine ; ce n'est pas une mansio, et les inscriptions qui s'y trouvent y ont été rassemblés, venant de la uia XXIV sur plusieurs milles : après le mille 9o, un milliaire au datif mentionnant Flavius Sévère en $306 / 307^{43}$, au $96^{\mathrm{e}}$ mille, à Aldehuela del Jerte un autre milliaire au datif, de Magnence (entre $35^{\circ}$ et 353$)^{44}$. Ce milliaire a été découvert à proximité de restes de sépultures ${ }^{45}$, indiquant la présence d'un noyau permanent d'habitations, à proximité d'une inscription funéraire ${ }^{46}$. En poursuivant sur quelques milles vers le nord, au mille 104 à Ahigal peut-être, un milliaire au datif de Maximien $^{47}$ en 305.

L'auteur de la notice de $H E p$ signale que la transcription est défectueuse, d'après la photographie, et qu'il est possible de compléter la restitution du texte, lecture reprise dans l' $A E$ : $[D$ (omino) $n$ (ostro $) I] m p$ (eratori) Fl $[$ a (uio) $] /[I]$ ulio Co $(n)$ sta $[$ nt $] i[o] /[$ Max $]$ imo triu $<m>f /$ ator $(i)[m] /$ axi $($ mo $)[s] e<m>$ per $A[u] / g u s($ to $), b($ ono $) r($ ei $) p($ ublicae $)[n($ ato $)]$.

43 CPILCaceres $712=$ MiliariosPlata $98=$ CILCaceres III 1108 Carcaboso: Imp(eratori) Caes(ari) F/lauio Val(erio) / Seuero / Inu(icto) Au[g(usto)] XC[---]. Pour Puerta Torres 1995, op. cit (n. 19), l'inscription aurait été découverte près du rio Jerte, puis fut transporté dans une finca : l'auteur la note comme étant à Galisteo.

44 J. Río-Miranda Alcón, <Inscripción de Aldehuela del Jerte 〉, BIGCV 6 (1981), 9 = RíoMiranda et Iglesias Domínguez 2005, op. cit. (n. 42), 9-10, n. 5, fig. $5(H E p$ 14, 81) = CILCaceres III 930 Aldehuela del Jerte: [D(omino) n(ostro) Magno ?] / Magne(n)/tio, uic/tori sem/per Augu/sto, bono / [rei] publi/cae nato / [---] ; autre lecture retenue par HEp : [---] / [d(omino) n(ostro) ---] / Magne(n/tio, uic/tori sem/per Aug (us)/to, bono / r(ei) $p($ ublico) $($ sic!) $n($ ato $)$.

45 J.E. Ortega, Corpus de inscripciones latinas de Caceres III. Capera (Cacéres 2013), 35 (=CILCaceres III $)$.

46 CILCaceres III 929 Aldehuela del Jerte, que l'on pourrait mettre en relation avec une éventuelle installation humaine.

47 J. Río-Miranda et $\mathrm{M}^{\mathrm{a}} \mathrm{G}^{\mathrm{a}}$ Iglesias Domínguez, « Nuevas aportaciones a la epigrafía y arqueología romana de Cáceres >, Ahigal 14 (2003), 4-5 (HEp 13, 211) = CILCáceres III 923 Ahigal : Imp(eratori) Caesar(i) M(arco) / [--- Va]lerio/ [---] Maxim(i)ano, / [pontif]<ici> maximo (sic!), / C[---] Imperatoris / [---] fortiissimo / Caesaris A(u)g(usto) / CIIII. Développé en $A(u) g$ (ustus) par les éditeurs et Ortega 2013, op. cit. (n. 45), il a été découvert en 2002 in situ. Le texte, outre les fautes de grammaire, propose une titulature qui pourrait être celle de Maximien et/ou de Maximin le Thrace et son fils. Il pourrait s'agir de la réutilisation d'un milliaire, peut-être martelé. 


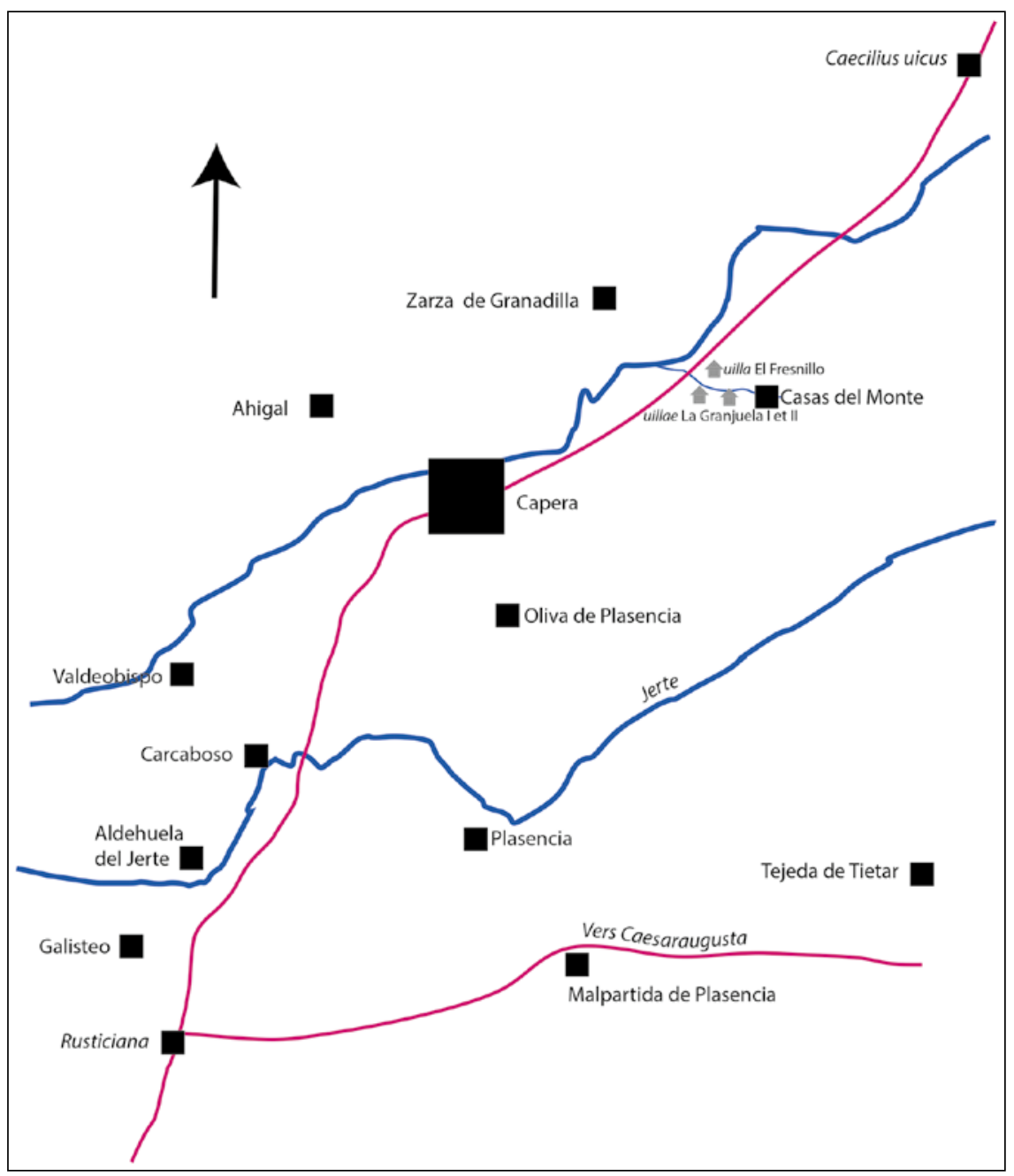

FIGURE 7.4 La uia XXIV aux environs de Capera

Capera, placée au centre de son territoire ${ }^{48}$, est aussi une des mansiones de la uia XXIV, et on y trouve donc des milliaires ${ }^{49}$ dont celui de Crispus au

48 Cerrillo Martín de Cáceres, Fernández Corrales et Herrera García de la Santa 199o, op. cit. (n. 16), 62, fig. 7 .

49 Itin. Anton. 433, 7 (ed. de Cuntz 199o, op. cit. [n. 15], 67); Ravenn. IV 45, 11: Cappara (ed. de Schnetz 199o, op. cit. [n. 25], 82). Cf. Roldán Hervás 1971, op. cit. (n. 14), 87-89. Pour les milliaires, par exemple: MiliariosPlata 10o: Nero Claudius Caes(ar) / Aug(ustus), pont(ifex) maxim(us), / trib(unicia) pot(estate) V, imp (erator) III, $p$ (ater) $p$ (atriae) / CII ; ERCCaceres 99 : Imp (erator) C(a)esa(r) /M(arcus) Aure[lius] / [---]IC[---], entre 276 et 282. 
datif ${ }^{50}$. À Oliva de Plasencia, on trouve un milliaire de Décence ${ }^{51}$ au datif. En s'éloignant de Capera, sans doute au $115^{\mathrm{e}}$ mille, et venant de Zarza de Granadilla, on trouve un milliaire au datif d'un empereur anonyme ${ }^{52}$.

Au nord du territoire de la cité de Capera, se trouve la petite ville de Casas del Monte, à proximité de la somptueuse uilla romaine de La Granjuela, une exploitation agricole ${ }^{53}$ implantée à l'est de la uia XXIV. Des bâtiments complémentaires, «La Granjuela II », ont été découverts lors de la construction de l'autoroute A 66. La uilla été détruite au $\mathrm{v}^{\mathrm{e}}$ siècle, réoccupée aux $\mathrm{VI}^{\mathrm{e}}-\mathrm{VII}^{\mathrm{e}}$ avec la présence d'un cimetière. Avec les grands travaux, d'autres découvertes ${ }^{54}$ ont été faites dans ce secteur, près du $118^{\mathrm{e}}$ mille de la uia XXIV, à $150 \mathrm{~m}$ de la confluence des rivières Garganta Grande et Ancha: la uilla «El Fresnillo II », en relation étroite avec une découverte plus ancienne qui pourrait être la pars urbana de cet ensemble qui constituerait la pars rustica ${ }^{55}$. Certains des petits bâtiments découverts le long de la voie pourraient être des tabernae diuersoriae.

On a découvert aux alentours des inscriptions funéraires ${ }^{56}$, des milliaires dont plusieurs au datif de Constance Chlore ${ }^{57}$ en 305, hélas sans milles indiqués ce qui renforce l'aspect < hommage public 〉, de Maximin Daia ${ }^{58}$ au mille

50 MiliariosPlata $112=$ CILCáceres II 1112 Segura de Toro $: D$ (omino) $N($ ostro $)[$ Fla $] /$ uio Iuli/o Cris $[$ po $] /$ nob(ilissimo) Caes(ari), / CX.

$51 \quad$ MiliariosPlata 113 = CILCáceres III 1077 Oliva de Plasencia : Dom(ino) n(ostro) / Magno / Decentio / nob(ilissimo) $C[$ aesari] / [---]. Lors de sa découverte dans le temple dédié à Jupiter, il a été considéré comme un hommage, mais sa forme est bien celle d'un milliaire. Décence est César en 350 et meurt en 353.

$5^{2}$ MiliariosPlata 120 = CILCáceres III 1074 Oliva de Plasencia ou Zarza de Granadilla : [---] / sem/per /Aug $[$ us $] /$ to u $[$ ic $] /[$ torimaxi (imo) $] /[C] X V$.

53 Elle nétait sans doute pas la seule le long de cet axe.

54 J. Rio-Miranda Alcón et Ma .G. Iglesias Domínguez, < Nuevos hallazgos arqueológicos en la A-66 >, article publié en-ligne : http://www.caparra.es/archivospdf/20-2004.pdf (consulté le $26 / 4 / 2019$ ).

55 Les découvreurs évoquent l'absence de «suntuosidad» dans les découvertes faites.

$5^{6} \quad$ CILCaceres III 962 Casas del Monte.

57 Río-Miranda et Iglesias Domínguez 2005, op. cit. (n. 42) 5-6, n. 2, fig. 2 (HEp 14, 88; FE 394) = CILCaceres III 964 Casas del Monte : [---] / [---] Cons $[$ tan $] /$ tio, $p($ io $)[f($ elici $)] / \operatorname{semp}($ er $)$ /Augus[to], / b(ono) $r(e i) p($ ublicae $) n(a t o)$. Il a été trouvé dans la villa «El Frenillo», en remploi.

$5^{8}$ CILCaceres III 965 Casas del Monte: [Im]p(eratori) Caes(ari) / Max(imin)o (sic!) / [---] RAIVS[---] / [ $p$ (ontifici) m(axim)]o, tri(bunicia) (sic!) / [po]tes(tate) / CXVIII. La présence d'un milliaire de Maximin Daia en péninsule Ibérique est sans doute liée à une décision de Constantin lui-même dans le cadre d'une période de concorde entre 305 et 310. Son nom aurait été ensuite martelé suite à la détérioration des relations entre les deux hommes. 
118, de Constantin I ${ }^{\text {er59 }}$ après 307 et avant 324 regravé sur le précédent, et un postérieur à $324^{60}$, un à un membre de la famille constantinienne ${ }^{61}$, qui pourrait être Constance II entre 324 et 337.

Depuis le nœud routier constitué par Rusticiana, part vers l'est une voie reliant Caesarobriga ${ }^{62}$. Sur son tracé, sur le territoire de la commune de Malpartida de Plasencia, dans la finca El Robledo a été découvert un milliaire de Constantin II César ${ }^{63}$ entre 317 et 337 : il est au datif, sans indication des milles. À ses côtés ce sont plusieurs inscriptions votives qui ont été découvertes ${ }^{64}-$ il $\mathrm{y}$ avait sans nul doute à proximité un lieu de culte au dieu Quangeius ${ }^{65}$ - ou de nature diverse ${ }^{66}$.

59 Río-Miranda et Iglesias Domínguez 2005, op. cit. (n. 42) 6, n. 3, fig. 3. = CILCaceres III 965 Casas del Monte : D(omino n(ostro) Fl(auio) V[alerio ---] / inuicto, / Augusto, / [---]N[---]us / nobilis(simo). $C[---] A[---] / O X[---] M A[---] ; C X V I I I$. Il est placé dans l'index par Ortega 2013, op. cit. (n. 45), 207, sous le nom de « Constancio I ».

6o CIL II $4670=$ CPILCaceres $714=$ MiliariosPlata $125=$ CILCaceres III 1107 Plasencia: $D D$ (ominis) $n$ (ostris )/ Flaui[o Va]l(erio) / [Constantino], / uicto[ri maximo] / semp $[$ er] / Aug $($ usto $) /[$ et ? ---]. Le document a été découvert dans un mur de la Plaza del Deán de Plasencia en remploi, mais il devait venir de la route reliant Casas del Monte et Segura de Tore. La fin du texte comprenait sans doute les noms des fils de Constantin.

61 MiliariosPlata 124 = J.-M. Solana Sainz et L. Sagredo San Eustaquio, La política viaria en Hispania. Signo IV D.C., (Valladolid 1997), 93, n. $21=$ ERCMCC 57, n. 47 = CILCaceres III 963 Casas del Monte: [--- Flauio Valerio ?] / Constan/tio, nobi/lissimo / Caesari / Brit(anico) / [---]. (Constance Chlore entre 296-305) mais il existe une autre lecture: [D(omino) $N($ ostro) ?] / [Flauio Iulio ?] / Constan/tio, nobi/lissimo / Caesari, / b(ono) r(ei) p(ublicae) $n($ ato $/$ [ [---]. (Constance II entre 324-337). Ce milliaire ne bornait pas la uia XXV Toletum-Caesarobriga-Augusta Emerita, mais bien la uia XXIV. La seconde hypothèse proposée dans la banque de données Hispania epigrafica (25511) et qui mentionne dans la bibliographie la première publication, ne tient absolument pas compte de la lecture des inventeurs, qui a pourtant été reprise ensuite. Le site ne fait que mentionner la variante possible Brit(anico) dont la lecture, sur la photo proposée, semble assez évidente, la troisième lettre apparaissant clairement comme un I et non un P. Nous préférons donc privilégier la proposition des inventeurs.

62 Pour Solana Sainz et Sagredo San Eustaquio 2006, op. cit. (n. 8), 115, ce milliaire appartient à la uia XXIV.

63 A. Sánchez Paredes, 〈Inscripciones latinas de la Vetonia〉, Diario Extremadura, $3^{1}$ de enero de 1964, n. $9($ ILER 1932) = CPILCaceres $745=$ MiliariosPlata 97, fig. 21, lám. $35=$ Solana Sainz et Sagredo San Eustaquio 1997, op. cit. (n. 61), 127, n. 118. = CILCaceres III 995 Malpartida de Plasencia: Caes[ari] / [Fla]uio / Clau/dio C/onst[a]/ntin/o Iun/iori, $n[o b($ ilissimo $)] / \mathrm{Ca}[$ es(ari)]. Il est possible qu'il appartienne à la uia XXIV, sans assurance, car une uia part de Rusticiana. On ne comprend pas l'introduction de $P(r) / o b o$ dans la restitution de Ortega 2013, op. cit. (n. 45), 83 .

64 Par exemple CILCaceres III 991 Malpartida de Plasencia $991 ; 992$.

65 CILCaceres III 991 Malpartida de Plasencia 993.

66 CILCaceres III 991 Malpartida de Plasencia 994: une funéraire; 996 : elle est illisible ; FE 588 : une funéraire? 
Sur le territoire de Capera, sur la voie en direction de l'est, au-delà de Tejeda de Tietar, on trouve à Berrocalejo, un milliaire de Maximien ou de Galère au datif ${ }^{67}$. Après ce rapide inventaire des milliaires de la uia XXIV, ce sont donc une dizaine de milliaires que nous pouvons isoler car au datif.

TABLEAU 7.1 Les milliaires au datif de la uia XXIV

\begin{tabular}{|c|c|c|c|}
\hline Nom du prince & Lieu de découverte & Date & Divers \\
\hline Caracalla & Mansio Turmulus & $212 / 217$ & \\
\hline Probus & Mansio Turmulus & $276 / 282$ & \\
\hline Maximien & Carcaboso & 305 & $\begin{array}{l}\text { Remploi après mar- } \\
\text { telage de Maximin le } \\
\text { Thrace? }\end{array}$ \\
\hline Flavius Sévère & Carcaboso & $306 / 307$ & \\
\hline Crispus & Capera & 317/326 César & $\begin{array}{l}\text { Entrée de la ville au } \\
\text { sud }\end{array}$ \\
\hline Constantin II & $\begin{array}{l}\text { Malpartida de } \\
\text { Plasencia }\end{array}$ & 317/337 César & \\
\hline $\begin{array}{l}\text { Constance Chlore } \\
\text { ou Constance II }\end{array}$ & Casas del Monte & 324/337 César & \\
\hline Constance II & Mansio Turmulus & $337 / 361$ & \\
\hline Magnence & Carcaboso & $35^{\circ / 353}$ & \\
\hline Décence & Capera & $35^{\circ} / 353$ & $?$ \\
\hline$?$ & Au nord de Capera & & \\
\hline
\end{tabular}

67 Milliaire de Maximien ou de Galère? Milliaire découvert à Berrocalejo, dans les ruines d'une forteresse musulmane, en remploi : cf. A. Moraleda Olivares, « Aportación al estudio de la epigrafía romana de la Extremadura oriental `, dans Centro de Estudios de los Montes de Toledo y la Jara (éd.), Homenaje a Fernando Jiménez de Gregorio (Tolède 1988), 97-107 = M. Santos Sánchez, La villa de Berrocalejo de Abajo, (Talavera de la Reina 1995), 98 = A. González Cordero, 〈Catálogo de inscripciones romanas del Campo Arañuelo, La Jara y Los Ibores >, dans D. Quijada González (éd.), VII Coloquios Históricos-Culturales del Campo Arañuelo. D. Justo Corchón García, (Navalmoral de la Mata 2001), 115-163, en part. 121-122, n. 7, avec photographie (HEp 11, 102): D(omino) n(ostro) / Maxi/miano, / nob(ilissimo) / Caes(ari); / XXXII (milia passuum). Variante : D(euotus) n(uminis) [maiestatisque eius] /Maxi/miano, / nob(ilissimus) (sic!) /Caes(ar) (sic!) / XXXII (milia passuum). Il serait daté de 285-286 s'il s'agit de Maximien, devenu Augustus en 286, ou de 293-305 s'il s'agit de Galère, qui est fait Augustus en 305. 


\section{Chronologie des milliaires au datif : la marque de moments clés?}

C'est tardivement que la titulature des empereurs sur les milliaires passe du nominatif au datif. Le texte concernant éventuellement Caracalla, venant de Garrovillas, ne peut être daté de ce règne : il faut bien entendu préférer y voir un milliaire de Probus ${ }^{68}$, le premier de notre chronologie, bien plus cohérent avec l'apparition des milliaires au datif. On peut donc, à mon sens, ne pas garder dans cet ensemble, un document, qui serait un milliaire, aujourd'hui perdu, au nom de Trajan, et au datif ${ }^{69}$ selon les éditeurs. Certes sa localisation, sur le tracé de la uia, pourrait en faire la borne du $31^{\mathrm{e}}$ mille. Mais l'état de la pierre rend douteux sa lecture effectuée au milieu du XIX ${ }^{\mathrm{e}}$ siècle.

Une fois l'habitude prise d'indiquer sur les milliaires le nom des princes au datif, c'est même à proximité du centre urbain que l'on va les trouver : l'un $^{70}$ est à placer entre 317 et 326 - il s'agirait de Crispus -, l'autre, aujourd'hui perdu, est daté de la première moitié $d u \mathrm{IV}^{\mathrm{e}}$ siècle $^{71}$.

Sur la via de la Plata, les derniers grands travaux ${ }^{72}$ semblent dater de Sévère Alexandre et de Maximin le Thrace. Ensuite, ce sont plutôt des interventions locales, sous Dèce ${ }^{73}$ en 250 et sous Probus ${ }^{74}$ en 282 et les milliaires de la Tétrarchie et des années 324-326 semblent davantage répondre à « un interés de propaganda dinástica ${ }^{75}$ » selon J.M. Roldán Hervás. Néanmoins rares sont les textes associant plusieurs membres de la famille impériale ; un seul milliaire

$68 C I L$ II 6204 $=$ CPILCaceres $683=$ MiliariosPlata $91=$ CILCaceres $\mathrm{I} 210(H E p 16,96)$ Garrovillas : Imp (eratori) Caes(ari) /Marco [Aur(elio) ---] / P(io) [F(elici)] Inuic/to Augu[sto], / [---], sur le pont d'Alconetar.

69 CIL II 4648 = CPILC 671 = MiliariosPlata 37 = CILCáceres I 69 Aldea del Cano : [---] / [---] Traiano [---] / [---] co(n)s(uli ?) II[---] / [---]. Cf. Roldán Hervás 1971, op. cit. (n. 14), 50, n. 18, qui ne donne pas le texte. Dans la base Hispania Epigraphica (21872), le texte a été donné au nominatif, selon la reconstruction <idéale > du Marquis de Monsalud: [Imp(erator) Caesar] /[diui Neruae filius], /[Nerua Tra]/ianus [Aug(ustus) Germ(anicus)],/[ $p]$ o(ntifex) $\max ($ imus $)$ tri(bunicia) $[$ pot(estate)], / $p($ ater $) p($ atriae $), c] o(n) s(u l) I I, /[$ restit $]$ uit. XXXI. Nous serions ici plus proche de la réalité.

70 MiliariosPlata $112=$ CILCáceres 1111112 Segura de Toro : $D$ (omino) $n$ (ostro) [Fla]/uio Iuli/o Cris $[$ po $] /$ nob(ilissimo) Caes(ari), / CX.

71 CILCáceres III 1076 Oliva de Plasencia:DD(ominis) nn(ostris) / F(l)auio / [I]u[lio - ].

72 Sillières 1990, op. cit. (n. 32), 595. Sur les travaux de restauration par Hadrien, cf. M ${ }^{a}$ P. González-Conde Puente, <La visita de Adriano a Hispania y la reparación de la vía de la Plata), Habis 51 (2020), 139-161, en part. 150-155.

73 Puerta Torres 1995, op. cit. (n. 19), 218-22o.

74 Puerta Torres 1995, op. cit. (n. 19), 221-222.

75 J.M. Roldán Hervás, 〈El camino de la Plata : historia de una vía romana〉, dans Sociedad Estatal de Commemoraciones Culturales 2008, op. cit. (n. 10), 41-47, en part. 46. 
au datif pourrait peut-être avoir compris Constantin et ses fils ${ }^{76}$, mais la partie inférieure manque. Les derniers milliaires connus, sous Décence et Valentinien ${ }^{{ }^{\mathrm{er}}}{ }^{\mathrm{ou}}$ Valens ${ }^{77}$ entre 354 et 367 , pourraient aussi relever de cette propagande.

La mise en place de ces milliaires au datif est liée à plusieurs moments: d'une part, aux alentours de $305^{78}$ avec Constance Chlore, Flavius Sévère, Maximien; d'autre part sous Constantin I ${ }^{\text {er }}$ Auguste et ses héritiers encore César avant 337, Constance II, Constantin II et Crispus chargé par son père Constantin des affaires occidentales - cela explique sans doute sa présence -; puis sous les successeurs de Constantin devenus Augustus avec Constance II ; enfin lors la crise de 350-353 avec Magnence et Décence César.

\section{$5 \quad$ Milliaires et hommages publics?}

Les voies constituent certes un lien entre les deux pôles qu'elles relient, animant le paysage rural et l'anthropisant. ${ }^{79}$ Mais elles sont en contact, sur tout leur tra-

76 CIL II $4670=$ CPILCaceres $714=$ MiliariosPlata $125=$ CILCaceres III 1107 Plasencia: $D D$ (ominis) $n$ (ostris) / Flaui $[$ o Va]l(erio) / [Constantino] / uicto[ri maximo] / semp $[$ er $]$ / Aug $($ usto $/$ [ et ? ---]. Le document a été découvert dans un mur de la Plaza del Deán de Plasencia en remploi, mais il devait venir de la route reliant Casas del Monte et Segura de Tore. La fin du texte comprenait sans doute les noms des fils de Constantin.

77 Un milliaire au nominatif, lacunaire : MiliariosPlata 75 Casar de Caceres.

78 Je voudrai signaler un texte qu'il faudrait sans doute joindre au dossier, un milliaire de Dioclétien découvert à Galisteo : il semble être à l'ablatif selon les développements proposés. Mais le milliaire ayant disparu, il faudrait peut-être revoir le développement et en faire un milliaire au datif, l'ablatif n'ayant ici pas de raison d'être : CIL II $4653=$ CPILCaceres 686 = MiliariosPlata $95=$ CILCaceres IV 1255 Galisteo $:[$ Im $]$ p(eratore) C [aesa $] /$ re Caio Val(erio) Dioc $[l e] /[t i]$ ano, $[p] i o,[f e l(i c i)], /$ inuict $[o, \operatorname{Aug}($ usto $)] /[---]$. Le texte est édité avec un développement à l'ablatif reposant en particulier sur $C[a e s a] / r e$. Le milliaire étant perdu, il est difficile de savoir si PERATORE à la ligne 1 figurait de façon développée ou si seul le P était visible ; l'édition la plus récente de Ortega 2013, op. cit. (n. 45), 109 est ainsi présentée : $[\operatorname{Im}] p$ (eratore). Mentionnant aussi la mention complète du prénom Caius à la ligne 2, anormale, alors que le gentilice Valerius est abrégé.

79 P. Sillières, < Les milliaires du sud de la péninsule Ibérique >, dans R. Étienne (éd.), Epigraphie hispanique. Problèmes de méthode et d'édition (Paris 1984), 270-281, en part. 272 : « Mais au IV ${ }^{\mathrm{e}}$ siècle, le changement est total : toutes les bornes sont au datif et ne différent donc en rien des simples dédicaces lorsque manque à la fin le chiffre des distances. Pourtant on ne peut douter qu'il s'agisse de milliaires lorsque les pierres proviennent des abords d'une voie antique »; 276 : «Mais au-delà du III ${ }^{\mathrm{e}}$ siècle, il serait aventuré de s'appuyer sur les milliaires pour connaître l'activité des chantiers, car un certain nombre d'entre eux ne sont probablement que des milliaires-dédicaces érigés comme de simples inscriptions honorifiques sans rapport avec de véritables travaux routiers ». M. Le Glay 
jet, avec les populations voisines, et permettent ainsi d'établir une relation entre les utilisateurs de la voie et les établissements qui la longent ${ }^{80}$. Le rôle des mansiones, des stationes, l'impact du cursus publicus ${ }^{81}$ était indéniable tant sur le plan économique, que social et culturel mais aussi politique. Ces liens établis au niveau local, avec les petits noyaux ruraux ou avec les uillae ${ }^{82}$ sont perceptibles dans l'utilisation des milliaires comme espace permettant de rendre hommage au prince, avec une titulature au datif. Ces relations s'établissent au niveau des grandes voies - on le voit bien dans la proximité avec la uia de la uilla de Santiago de Bencáliz ${ }^{83}$, près de la mansio Ad Sorores ${ }^{84}$ situé au $26^{\mathrm{e}}$ mille, mais sans doute aussi au voisinage des routes secondaires, utilisées par les populations locales. C'est là que l'impact du milliaire-dédicace est sans doute le plus fort, car c'est régulièrement que les occupants des uillae et autres domaines passent devant ces milliaires et utilisent les voies pour écouler leurs productions.

Or, dans le cadre du dossier de la uia XXIV, les centres poliades sont rares, et l'espace territorial de chacune de ces cités est assez vaste, comme on le constate pour Capera ${ }^{85}$. Le contact avec les centres civiques, les forums où se dressent normalement les hommages rendus aux princes est donc minoré par

dans la discussion sur cette communication (291) proposait que « dans les dédicaces au datif entre une part de préoccupation religieuse », en y voyant une manifestation du culte impérial. P. Sillières (293) a répondu qu'effectivement ces milliaires sont des «instruments de la propagande impériale», en particulier pour « ces monuments découverts en groupe et élevés à des dates très rapprochées » - il parle de «nids de milliaires ». Il ajoute que «ces inscriptions manifestent l'adhésion des populations à un nouveau prince et constituent une forme nouvelle du culte impérial », mais plus tardivement qu'en Afrique : en péninsule Ibérique, les milliaires au datif apparaissent au $\mathrm{IV}^{\mathrm{e}}$ siècle. Cette idée est reprise par Roldán Hervás 2008, op. cit. (n. 75), 46.

8 o S. Haba Quirós et V. Rodrigo López, «La vía de la Plata entre las mansiones Rusticiana y Caecilius Vicus: la calzada en relación con el asentamineto>, dans Institución Fernando el Católico (éd.), Simposio sobre la red viaria en la Hispana romana (Saragosse 1990), 241-252, en part. 244.

81 H.-G. Pflaum, < Essai sur le cursus publicus dans le Haut Empire 〉, Mémoires présentées par divers savants à l'Académie des inscriptions et belles-lettres de l'Institut de France 14 (1940), 189-391 ; A. Kolb, Transport und Nachrichtentransfer im Römischen Reich (Berlin 200o).

82 Gorges 199o, op. cit. (n. 4), 91 : « La confrontation de l'implantation rurale romaine avec le réseau de communication achève de donner leur importance à ces secteurs ».

83 E. Cerillo Martin de Cáceres, « Santiago de Bencaliz. Un asentamiento rural en la vía de la Plata >, NAH 13 (1982), 165-212 : la villa aurait deux périodes de construction : au I ${ }^{\mathrm{er}}$ siècle p.C. puis au $\mathrm{IV}^{\mathrm{e}}$ siècle, avec une complète transformation.

84 V. Gil Mantas, As Vias Romanas da Lusitânia (Mérida 2012), 296-297, fig. 101.

85 Cerrillo Martín de Cáceres, Fernández Corrales et Herrera García de la Santa 199o, op. cit. (n. 16), 57 , fig. 3 ; ils ont utilisé pour cet article les polygones de Thiessen, en reconnaissant les limites de cette méthode. 


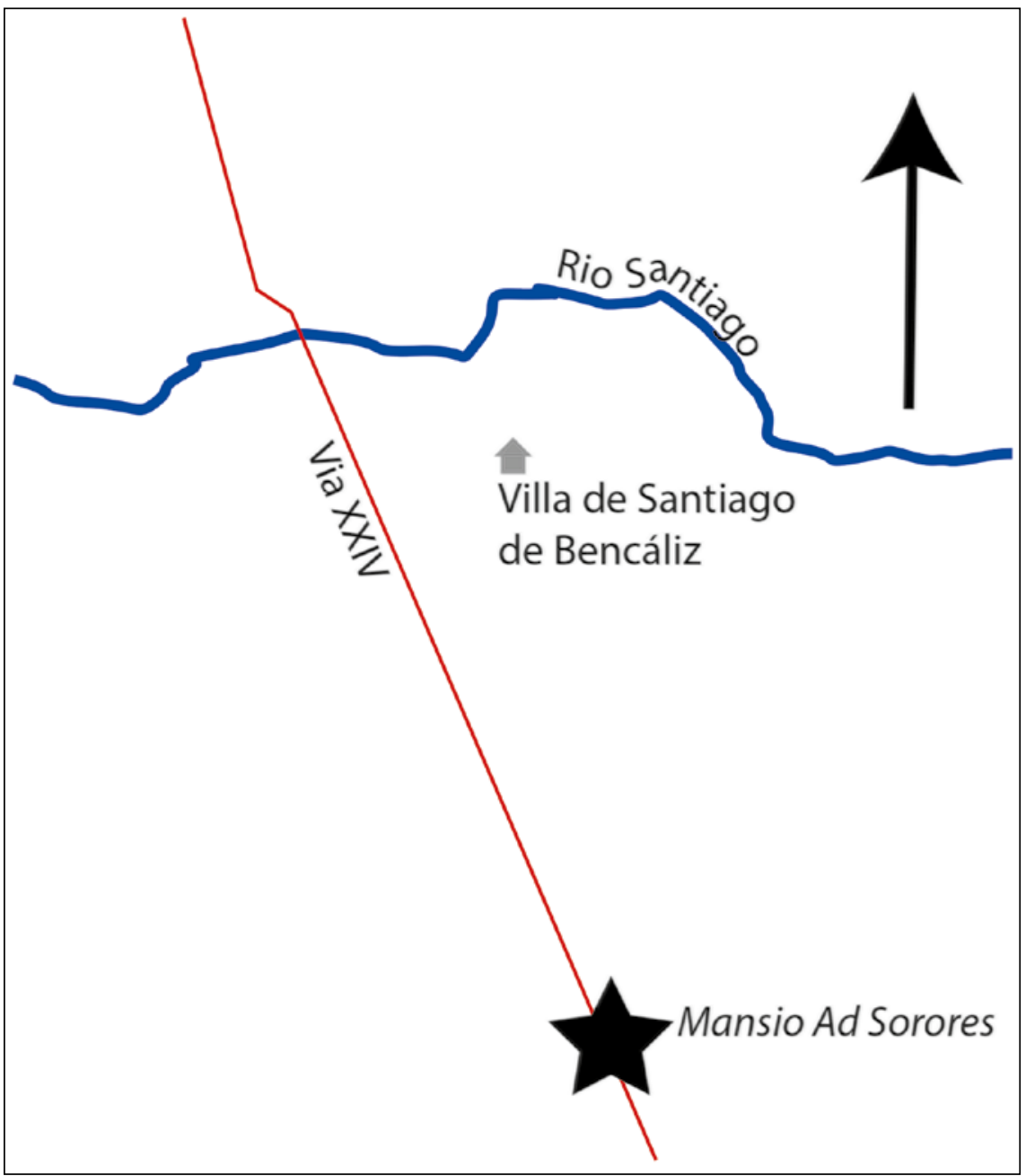

FIGURE 7.5 Les environs de la mansio ad Sorores

rapport à ce que peuvent vivre les citoyens d'Augusta Emerita ou d'autres cités, situées plus au sud, en Bétique ${ }^{86}$. Mais ces hommages publics, décrétés normalement par les cités, semblent avoir été rares $^{87}$ : à Norba, fondation césarienne,

86 Cerrillo Martín de Cáceres, Fernández Corrales et Herrera García de la Santa 199o, op. cit. (n. 16), 57 , fig. 3 : on constate une nette différence entre la taille des cités de l'est de la Lusitanie et du Nord de la Bétique.

87 En fonction de la documentation connue à ce jour. 
un seul hommage est connu de façon sûre ${ }^{88}$, rendu à Septime Sévère en $194^{89}$. Ce piédestal de marbre était surmonté d'une statue ou statuette en argent de dix livres, l'ordinatio du texte est tout à fait remarquable ; si la décision est clairement prise par l'ordo de la cité de Norba, on peut se demander qui en est à l'initiative ${ }^{90}$. Un autre document ${ }^{91}$ pourrait être un hommage rendu au prince, à Trajan; il a été découvert au centre de la ville de Cáceres au $\mathrm{XVIII}^{\mathrm{e}}$ siècle - il est aujourd'hui perdu -; compte tenu des lettres qui restaient lisibles, il est difficile de dire si le texte était au nominatif - certains ont pensé qu'il pourrait s'agir d'un milliaire -, ou au datif. S'il était possible de restituer Germanicus et pontifex maximus en entier, on pourrait avoir des doutes sur un support milliaire ; la découverte au centre de la cité conduit plutôt à y voir un hommage qu'il faudrait alors proposer au datif ${ }^{92}$. La ville de Caurium, qui n'est pas située sur le tracé principal de la Via de la Plata, pas plus que son territoire, n'a pas gardé d'hommages rendus aux princes.

Quant à Capera ${ }^{93}$, elle apparaît comme étant une cité modeste qui, appartenant au territoire vetton, était stipendiaire à l'époque augustéenne, puis a été dotée du droit latin et est devenue municipe sous les Flaviens ${ }^{94}$. C'est une toute petite ville ${ }^{95}$, enceinte d'une muraille dont on ignore la date de construction ${ }^{96}$. Les hommages publics rendus à la famille impériale y sont rares : on ne peut en

88 Le dossier du pont d'Alcántara et du temple voisin est évidemment à mettre à part : trois hommages aux princes en proviennent: CILCáceres I 18; 20 (avec des doutes importants sur l'exactitude de cette copie moderne) à Trajan ; 21 à Antonin le Pieux.

$89 \quad$ CILCaceres I 113 Norba.

90 S. Lefebvre, <Les hommages rendu aux princes dans les trois provinces de péninsule Ibérique : uniformité ou différences de comportement des dédicants? >, dans A. Gangloff (éd.), Les miroirs aux princes, à paraitre.

$91 \quad$ CIL II $692=$ CPILCaceres $103=$ CILCáceres I 115 Cáceres: [Imperator Cae]sar / D(iui $)$ Nerua[e f(ilius)] / [Nerua Traianus] Aug(ustus) G[ermanicus], / [pontifex maximus] trib(unicia) $p$ (otestate) / [---].

92 Sous cette forme: [Imperatori Cae]sar(i) / D(iui) Nerua[e f(ilio)] / [Neruae Traiano] Aug (usto) G[ermanico], / [pontifici maximo], trib(unicia) p(otestate) / [---].

93 J. Río-Miranda Alcón, La ciudad romana de Cáparra, municipium Flavium Caparense, (Pampelune 2010).

94 E. Cerrillo Martín de Cáceres, <Capara, municipio romano >, dans J.-G. Gorges et T. Nogales Basarrate (éds.), Sociedad y cultura en Lusitania romana (IV Mesa Redonda Internacional, Mérida) (Mérida 2000), 155-164.

95 J.M. Blázquez, Caparra. Excavaciones arqueologicas en España 34 (Madrid 1965), 13. On estime sa surface à 14 à 16 hectares.

96 Blázquez 1965, op. cit. (n. 95), 13 donne le $\mathrm{III}^{\mathrm{e}}$ siècle; mais L. Brassous, Les villes de la péninsule Ibérique au III siècle p.C. (Thèse doctoral, Bordeaux 2010) II, 807, a repris le dossier et signale que l'enceinte en opus quadratum pourrait être bien antérieure. 
mentionner qu'un seul, un hommage dynastique rendu à Iulia Domna ${ }^{97}$ sans doute peu après 197 ; d'autres documents sont trop abîmés pour qu'ils puissent fournir des informations ${ }^{98}$. Il semble qu'au début du IV siècle, les espaces publics soient en partie privatisés ${ }^{99}$ : les lieux privilégiés où devraient se trouver les hommages rendus aux princes perdent leur physionomie monumentale et leur prestige. Ce sont d'autres lieux qui vont accueillir les inscriptions honorifiques, en particulier dans les espaces peu urbanisés mais où les uillae jouent un rôle important ${ }^{100}$.

Avec une population sans doute plus habituée à voir le nom des princes sur les milliaires le long des voies que sur les forums des villes, les utiliser comme support pour rendre hommage à l'empereur a pu apparaître comme une solution peu coûteuse et efficace. Ils faisaient partie du paysage vécu par les ruraux.

Néanmoins, c'est alors toute une partie de la propagande impériale qui va être mise de côté : moins de place pour développer la titulature, moins de place pour faire figurer les héritiers potentiels, absence d'une statue couronnant habituellement l'hommage public en milieu urbain, alors que l'usage du milliaire au datif se développe dans un contexte de crises politiques au sommet de l'État. Ainsi que l'évoque S. Benoist ${ }^{101}$, la rhétorique du discours impérial passe d'une part par des documents normatifs, mais aussi par des supports qui relaient les hommages traditionnels.

C'est sans doute pour cela que l'on voit sur ces milliaires le nom des Césars tout aussi présent que celui des Augustes: Crispus, Constance II et Constantin II encore César tous trois. Dans ces trois milliaires, le nom de leur père n'est pas mentionné, et leur titre de nobilissimus Caesar est bien présent. Le prince régnant, Constantin I ${ }^{\mathrm{er}}$ n'est pas honoré, mais ce sont ses trois fils associés au pouvoir qui le sont.

97 CIL II $810=$ CPILCaceres $185=$ CILCaceres III 1012 Capera: Iuliae Aug(ustae) matri castror(um), / coniugi Imp(eratori) Caesaris L(uci) Sept(imi) / Seueri pii Pertinacis Aug(usti) et matri M(arci) Aur(elii) Ant(onini) Imp(eratoris) / [ [et P(ublii) Sept(imi) Getae nob(ilissimi) Caes(aris) ?]], / ordo splendidis[simus] / Cap[erensium deuotus / numini maiestatique eius].

98 CIL II $811=$ CPILCaceres $189=$ CILCaceres III 1063 Capera : [--- Ca $]$ esari $/$ C[---]em/o [---]nis; CPILCaceres 687 : [--- tribunicia pot] estas [---]/[---]pro.

99 Cerrillo Martín de Cáceres 200o, op. cit. (n. 94), 163; Ortega 2013, op. cit. (n. 45), 13. Pour une étude plus globale, cf. P. Diarte Blasco, < La convivencia de lo público y lo privado. El establecimiento de unidades domésticas y artesanales en los espacios cívicos hispanos >, dans L. Brassous et A. Quevedo (éds.), Urbanisme civique en temps de crise. Les espaces publics d'Hispanie et de l'Occident romain entre le II e et le IV ${ }^{e}$ siècle (Madrid 2015), 289-307.

100 Gorges 199o, op. cit. (n. 4), 93.

101 S. Benoist, < Identité du prince et discours impérial : le cas de Julien >, AntTard 17 (2009), 109-117, en part. 112. 
En travaillant sur la province de Bétique, P. Sillières ${ }^{102}$, à la suite de P. Salama pour l'Afrique ${ }^{103}$, a proposé de voir dans ces milliaires où la titulature est au datif des dédicaces ${ }^{104}$; leur rôle de milliaires, de marqueur de l'espace y est peu présent avec l'absence souvent des distances ${ }^{105}$, quand on a la chance d'avoir les milliaires complets. Ils ne servent plus alors à mesurer l'espace, à dimensionner le paysage rural. L'initiative impériale manque aussi souvent: les verbes habituellement trouvés sur les milliaires, fecit, refecit ou restituit ${ }^{106}$ sont absents. On constate aussi que sur certains tronçons, voire sur le même site, on trouve des milliaires très proches dans le temps, qui ne peuvent être liés à des réfections de la voie ${ }^{107}$. Comme le propose P. Sillières ${ }^{108}$, on peut voir dans ces milliaires-hommages au datif la manifestation de «l'adhésion des populations à un nouveau Prince » et peut-être «une forme nouvelle du culte impérial ${ }^{109}$ » dans des régions où les dédicaces impériales sont effectivement totalement absentes des centres urbains de moyenne taille en ce début du IV e siècle, ce qui semble être le cas dans les capitales de cité liées à la uia XxIv. Selon sa formule ${ }^{110}$, «la voie relaie désormais le forum». C'est dans un espace non pas nouveau mais dont

102 P. Sillières, <Les milliaires du sud de la Péninsule ibérique >, dans Étienne 1984, op. cit. (n. 79), 270-281, en part. $272 ; 292$ dans la discussion ; P. Sillières, « De la borne milliaire à la dédicace impériale. L'exemple de quelques inscriptions de l'Hispanie méridionale `, REA 88 (1988), 351-358, en part. $35^{\prime} ; 35^{2}$.

103 P. Salama, < La colonie de Rusguniae d'après les inscriptions >, Revue Africaine 99 (1955), 5-52, en part. 22: «Ces milliaires sont par conséquent des dédicaces dont la colonie de Rusguniae voulait honorer les souverains, et les bornes elles-mêmes des monuments de dévotion ... » ; 24. Cf. aussi I. König, ‘ Zur Dedikation römischer Meilensteine. Digesta, 43, 7, $2 ;$ 50, 10, 3-4 >, Chiron 3 (1973), 419-427.

104 J. Arce, < Los miliarios tardorromanos de Hispania : problemática histórica y epigráfica >, dans Étienne 1984, op. cit. (n. 79), 289-294, en part. 289.

105 J. Arce, « Epigrafía de la Hispania tarorromana de Diocletiano a Teodosio : problemas de historia y de cultura >, dans A. Donati (éd.), La terza età dell'epigrafia (Atti del convegno); colloquio AIEGL, Borghesi 86 (Bologna 1986) (Faenza 1988), 211-227, en part. 224: « miliarios, no siempre mensurativos, sino propagandísticos ».

106 J.-M. Solana Sainz et L. Sagredo San Eustaquio, < La política edilicia viaria imperial en la Hispania del s. IV d.C. >, dans R. Teja et C. Pérez Gonzalez (éds.), Actas : Congreso Internacional La Hispania de Teodosio (Valladolid et Segovia 1997), 255-274, en part. 255.

107 P. Sillières, < Un grupo de quatro miliarios en La Cerradura (Pegalajar, Jaén) >, Bolletin del Instituto de Estudios Giennenses 90 (1976), 55-70 : un milliaire de Maximin Daia, un milliaire de Constantin, un milliaire de Crispus.

108 Sillières 1984, op. cit. (n. 102), 293 dans la discussion.

109 Si je suis P. Sillières pour les milliaires-hommages, je suis moins convaincue par sa proposition d'y voir une forme nouvelle du culte impérial, en raison de l'absence de milliaires-hommages aux diui entre autres.

Sillières 1988, op. cit. (n. 102), 351; $35^{2}$. 
l'utilisation évolue à la fin du $\mathrm{III}^{\mathrm{e}}$ et au début du $\mathrm{IV}^{\mathrm{e}}$ siècle que l'impact de Rome se fait désormais sentir : le paysage des espaces ruraux se politise.

\section{$6 \quad$ Conclusion}

Ce dossier est un témoignage de la variété de l'impact de Rome dans les espaces ruraux, loin du centre urbain de la ciuitas. L'exemple utilisé, celui de la uia xxiv permet d'appréhender la problématique, d'essayer de l'analyser dans un cadre spatial relativement limité, mais il ne répond pas à plusieurs questions, dont la principale: qui a eu l'idée de cette évolution du milliaire-borne au milliaire-hommage? Qui a acté le passage du nominatif au datif? Qui, ensuite, prenait la décision d'implanter ces milliaires-hommages le long des routes, souvent sans mentionner le nombre de milles? Si le nord de la Lusitanie permet cette analyse sur un tronçon de la uia XxIv, il ressort de sondages ponctuels effectués dans tout l'empire, que l'on retrouve cette pratique aussi bien en Afrique, comme l'avait déjà signalé Pierre Salama ${ }^{111}$, mais aussi en Orient ${ }^{112}$. Des allusions ont pu être faites ${ }^{113}$, mais aucune étude systématique sur l'ensemble de l'empire n'a été à ma connaissance menée ${ }^{114}$.

Le dossier lusitanien permet cependant de proposer une première étape de la réflexion : les voies sont du ressort de l'empereur ${ }^{115}$, ses ordres sont appliqués par les hauts fonctionnaires provinciaux; c'est donc bien du pouvoir central qu'émane l'idée de mettre en place ces milliaires-dédicaces. Mais il ne s'agit alors pas d'un véritable hommage, en dépit de la présence du datif, tel que les communautés pouvaient le rendre au prince, de façon assez libre et autonome ; il n'est en effet pas de leur fait, et n'est pas un témoignage de la popularité de tel ou tel prince ${ }^{116}$. Il consacre au contraire l'autorité impériale, y compris dans des moments de crise. Le milliaire-dédicace est une forme de l'affirmation de l'autorité impériale, affichée dans des lieux stratégiques, le long des voies, dans un cadre non monumentalisé comme pouvait l'être un forum; son texte au datif évoque l'hommage public pour des populations qui ont pu voir de vrais hommages au centre des cités. Mais il n'en est pas réellement un. En prenant place dans le paysage, il est cependant une forme d'impact de Rome que l'on ne peut négliger.

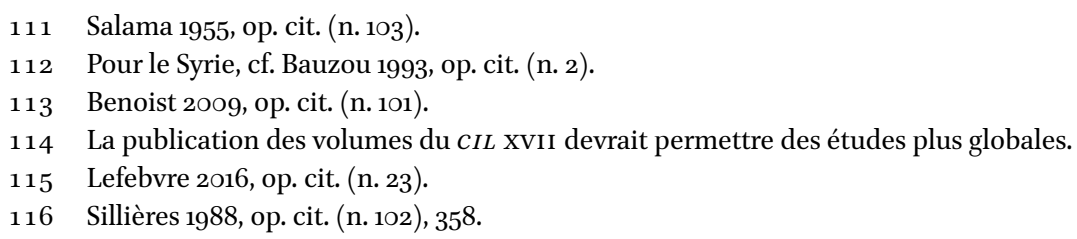




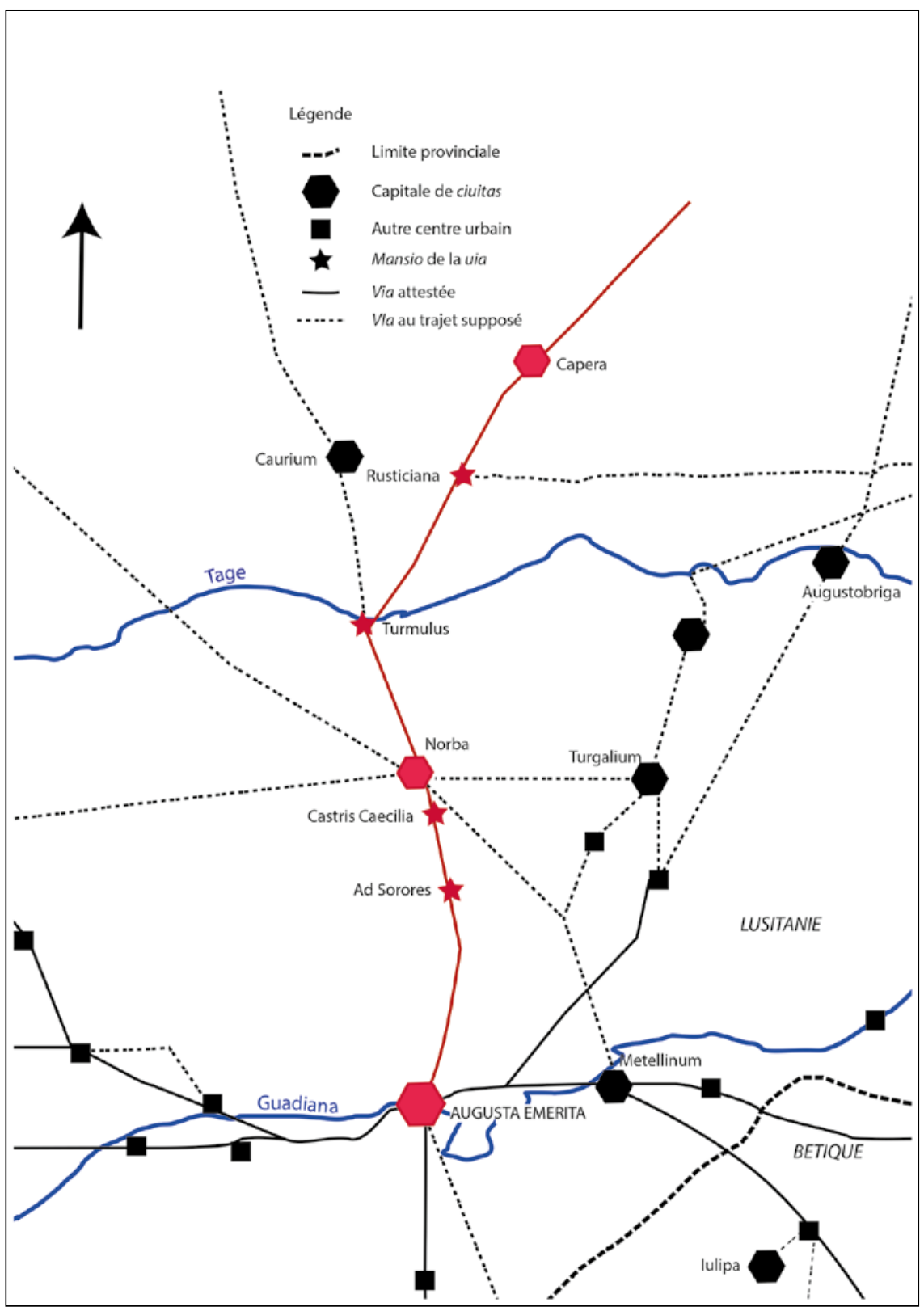

FIGURE 7.6 La section lusitanienne de la Via de la Plata 